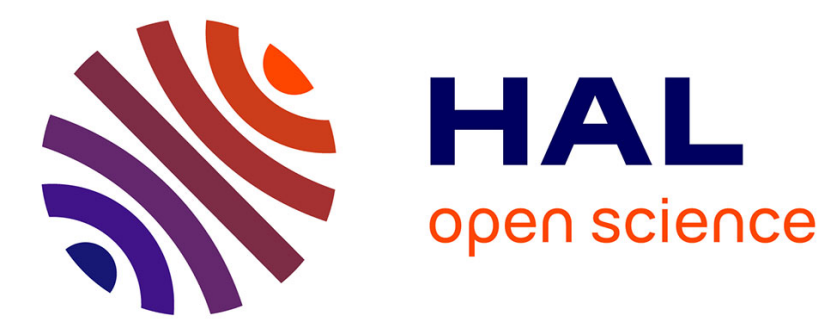

\title{
Les migrations internes accentuent l'inégalité historique du peuplement de la Chine
}

Gérard-François Dumont, Yiliminuer Tuerxun

\section{To cite this version:}

Gérard-François Dumont, Yiliminuer Tuerxun. Les migrations internes accentuent l'inégalité historique du peuplement de la Chine. Informations sociales, 2014, 185, p.24-32. 10.3917/inso.185.0024 . halshs-01099534

\section{HAL Id: halshs-01099534 https://shs.hal.science/halshs-01099534}

Submitted on 4 Jan 2015

HAL is a multi-disciplinary open access archive for the deposit and dissemination of scientific research documents, whether they are published or not. The documents may come from teaching and research institutions in France or abroad, or from public or private research centers.
L'archive ouverte pluridisciplinaire HAL, est destinée au dépôt et à la diffusion de documents scientifiques de niveau recherche, publiés ou non, émanant des établissements d'enseignement et de recherche français ou étrangers, des laboratoires publics ou privés. 


\section{Les migrations internes accentuent l'inégalité historique du peuplement de la Chine}

Gérard-François Dumont - géographe, économiste et démographe Tuerxun Yiliminuer - géographe

L'immense territoire de la Chine a toujours été peuplé de façon très inégale, la moitié Sud-Est concentrant la quasi-totalité de la population. Au cours du XX $X^{\mathrm{e}}$ siècle, des politiques migratoires changeantes ont donné lieu à des déplacements massifs de population, dans un sens ou dans un autre. Aujourd'hui, la pauvreté des régions rurales et l'inégalité de statut entre les villes et les campagnes, ainsi que les stratégies matrimoniales qu'elles suscitent, renforcent le déséquilibre.

Dans l'immense pays qu'est la Chine avec ses 9,7 millions de $\mathrm{km}^{2}$, soit dix-huit fois la superficie de la France métropolitaine, les conditions géographiques, climatiques, historiques, économiques et sociales ont inévitablement engendré de grandes différences de peuplement selon les territoires. Les écarts structurels de peuplement ont évolué différemment au fil de la politique changeante du parti communiste vis-à-vis des migrations internes. Le seul aspect pérenne dans cette politique est le souci du pouvoir de développer le peuplement d'ethnie Han dans des régions où cette dernière a toujours eu un poids démographique marginal, voire nul. Depuis les réformes économiques entamées en 1978, l'émigration rurale massive crée de nouvelles inégalités en raison du statut inférieur de la population d'origine rurale vivant en ville. Une partie de cette émigration rurale est également due à des stratégies matrimoniales en réponse aux inégalités sociales.

Chine du " plein » et Chine du « vide»

Depuis 1935 au moins, l'habitude a été prise de présenter le peuplement du pays en différenciant deux parties. Cette année-là, le géographe Huanyong Hu trace une diagonale séparant la Chine en deux en distin- 
guant la partie Sud-Est et la partie Nord-Ouest. À cette époque, la première, la Chine du "plein ", où dominent les plaines et les bassins des fleuves principaux issus des montagnes occidentales, concentre $96 \%$ de la population totale sur $36 \%$ de la superficie tandis que la Chine du "vide », dans le Nord-Ouest, où dominent montagnes et plateaux, ne compte que $4 \%$ de la population sur $64 \%$ de la superficie totale du pays. Quatre-vingts ans plus tard, cette dualité persiste avec un changement marginal dans les proportions : le Sud-Est compte $94 \%$ de la population contre $6 \%$ dans le Nord-Ouest.

Même si cette dualité se perpétue, la réalité du peuplement de la Chine doit toutefois être appréhendée plus finement. II convient ainsi de distinguer les trente et une entités administratives du territoire chinois soit, plus précisément, vingt-deux provinces, cinq régions dites autonomes (même si leur autonomie réelle est bien faible, comme dans le Xizang au Tibet) et quatre grandes agglomérations : Pékin, Shanghai, Tianjin et Chongqing, dont les municipalités, dites "particulières » ou " de rang provincial ", relèvent directement du gouvernement central (1). Parmi ces quatre municipalités, Shanghai et Tianjin disposent d'un port très important (respectivement les premier et troisième ports mondiaux en volume de marchandises), Pékin est la capitale politique tandis que Chongqing, était, avant de bénéficier de ce statut, I'une des principales villes de la province intérieure du Sichuan. Cinq des trente et une entités administratives dépassent chacune $550000 \mathrm{~km}^{2}$ de superficie, soit la superficie de la France métropolitaine : le Xizang (Tibet), le Xinjiang, le Sichuan et le Qinghai, situées dans l'Ouest de la Chine, ainsi que la Mongolie intérieure, au Nord de la Chine.

\section{Des écarts considérables de peuplement}

Selon le sixième recensement, effectué en 2010, la population de la Chine atteint 1,333 milliard d'habitants, avec des répartitions très variables dans chaque entité administrative. Les trois les plus peuplées comptent un peu plus ou un peu moins de cent millions d'habitants, soit davantage que la population du pays le plus peuplé de l'Union européenne, I'Allemagne. Il s'agit, par ordre décroissant, du Guangdong, province maritime de l'extrême Sud-Est, dont le littoral avoisine Macao et Hong Kong, et de la province également maritime du Shandong, dans le Centre-Est ; la troisième entité, proche de 100 millions d'habitants, est le Henan au cœur de la plaine centrale traversée par le fleuve Jaune, le principal fleuve de la Chine du Nord, et donc à fort potentiel agricole.

À l'opposé, quatre entités administratives comptent moins de dix millions d'habitants. Le faible peuplement de deux d'entres elles peut s'expliquer par leur superficie réduite : il s'agit de l'île de Hainan, au sud de la Chine, et de la région autonome du Ningxia. En revanche, les deux autres 
sont deux des trois plus vastes entités administratives de l'ouest du pays : le Xizang, dont le nom officiel est "région autonome du Tibet », et le Qinghai.

Au plan de la densité, la Chine compte 140 habitants au $\mathrm{km}^{2}$, un chiffre supérieur de $20 \%$ à celui de la France mais inférieur à celui de I'Allemagne ou de l'Italie. II n'est pas surprenant de trouver les densités les plus élevées dans les quatre municipalités de rang provincial. Shanghai, dont la superficie équivaut à celle d'un département français, avoisine 3800 habitants au km², devançant Pékin qui compte 1167 habitants au km² et Tianjin, 1145 habitants au km². Ces deux dernières ont donc une densité de population à peine supérieure à celle de la région Île-de-France. Les quatre autres provinces dont la densité dépasse 500 habitants au $\mathrm{km}^{2}$ forment un demi-cercle, allant de la province littorale du Zhejiang à celle du Henan en passant par deux autres provinces littorales, le Jiangsu et le Shandong. Outre la municipalité de rang provincial de Chongqing, les sept provinces comptant entre 301 et 500 habitants au $\mathrm{km}^{2}$ sont toutes situées dans la Chine du «plein ».

Carte 1. La densité de la population en Chine (nombre d'habitants au $\mathbf{k m}^{2}$ )

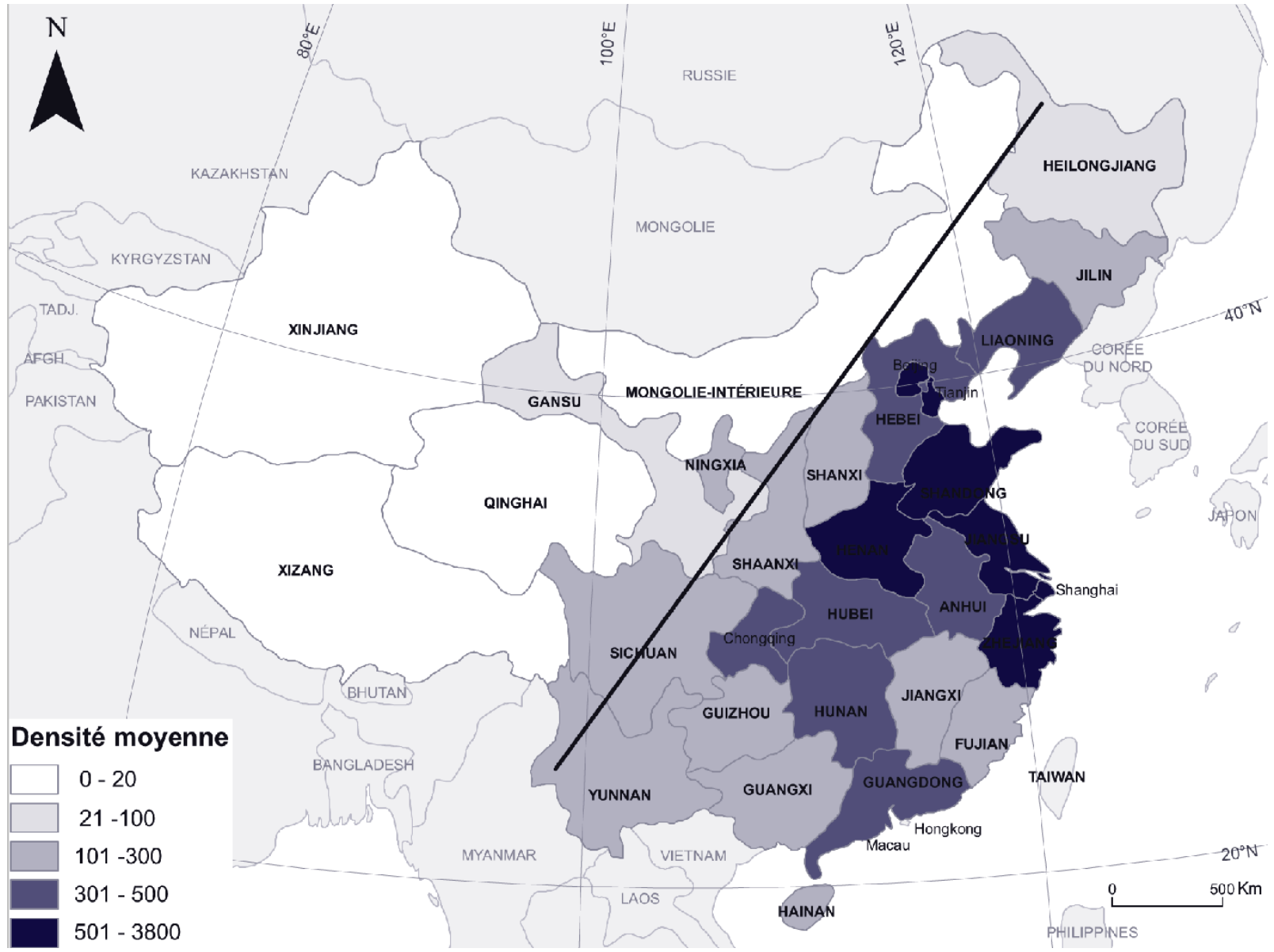

C Population et Avenir, chiffres BNSC 2012. 
Deux d'entre elles, le Liaoning et le Hebei, sont limitrophes ou proches des municipalités de rang provincial de Pékin et Tianjin. Les cinq autres dessinent un arbre qui s'étend en continuité des provinces les plus denses, dont les racines seraient la province maritime de Guangdong. Dans les huit provinces dont la densité moyenne est comprise entre 101 et 300 habitants au $\mathrm{km}^{2}$, le peuplement est souvent très contrasté car la population se concentre dans des deltas, des vallées ou des bassins.

Enfin, aux niveaux de densité les plus bas, quatre entités administratives de la Chine du "vide", Mongolie intérieure, Qinghai, Xinjiang et Xizang, comptent moins de 20 habitants au $\mathrm{km}^{2}$ : c'est la Chine des montagnes et/ou des terres arides. Toutefois, le peuplement de ces provinces peut également s'avérer très concentré sur de petites portions de leurs territoires.

Si le poids démographique relatif des territoires du nord-ouest de la Chine a toujours été faible, il a toutefois légèrement augmenté pour deux raisons : une politique de limitation des naissances moins stricte pour les ethnies minoritaires $\boldsymbol{\zeta}$ (...) le phénomène des migrations qui (...) favorisent les régions littorales et les deux provinces les plus occidentales. l'évolution du peuplement en Chine, de prendre en compte le phénomène des migrations qui, pour simplifier, favorisent les régions littorales et les deux provinces les plus occidentales. Mais avant qu'il en soit ainsi, les directives du Parti communiste chinois concernant les migrations internes ont beaucoup évolué entre autorisation, interdiction, obligation ou laisser-faire.

\section{Des politiques migratoires changeantes}

Entre 1949 et 1957, les Chinois peuvent librement choisir de changer de territoire de domicile. Le gouvernement, souhaitant développer l'économie urbaine, encourage alors la migration spontanée vers les villes. En 1953, le premier plan quinquennal est mis en place. Il donne la priorité à l'envoi de jeunes actifs de la zone littorale, la plus peuplée, vers la province de l'extrême nord-est, le Heilongjiang, et vers la région autonome du nord-ouest, le Xinjiang. Cette migration économique, qui vise à accroître la présence d'actifs pour l'exploitation du potentiel industriel du Heilongjiang et celle de nouvelles terres dans les régions frontalières du nord-ouest, additionne une migration spontanée et une migration organisée par l'État. Ce dernier y voit en effet un moyen d'augmenter la proportion des Chinois de l'ethnie Han dans des régions qui sont respectivement mandchoue et ouïghoure. À compter de 1955, cette politique s'applique au Tibet. La période 1958-1965 commence avec une nouveauté administrative, l'instauration en 1958 du système du livret de résidence 
(hukou), une sorte de passeport intérieur n'autorisant que les migrations internes acceptées par l'administration. Le gouvernement, considérant alors qu'il faut dé-densifier les villes, organise des migrations forcées vers les campagnes en application du deuxième plan quinquennal (1958-1962). Ces migrations forcées se doublent de migrations spontanées en raison des conséquences de la politique du "Grand Bond en avant ", c'est-àdire de l'intensification de la collectivisation. Celle-ci se traduit par une terrible famine engendrant une considérable mortalité pendant ce que l'on appelle les «trois années noires » (1959 - 1961). Pour échapper à la famine, des habitants de l'Est et du Centre émigrent spontanément vers le Heilongjiang, le Xinjiang ou la Mongolie intérieure.

Entre 1962 et 1965, en raison de la pénurie de matières premières, nombre d'usines situées dans les zones urbaines sont fermées. Le gouvernement préconise alors le retour à la campagne des travailleurs urbains et des migrants retournent dans leur région d'origine.

La période suivante, 1966-1977 est celle de la Révolution culturelle, pendant laquelle le gouvernement applique strictement le système d'enregistrement des ménages, empêchant toute migration de la campagne vers la ville. Qui plus est, pour limiter l'urbanisation, il envoie à la campagne environ quinze millions de jeunes diplômés et nombre de travailleurs urbains.

\section{Une émigration rurale massive}

Les réformes économiques entamées en 1978 modifient totalement la donne. Elles se traduisent par un relâchement du contrôle des migrations internes qui donne lieu à une émigration rurale massive évoquant le proverbe chinois: "L'eau s'écoule vers le bas; l'homme, lui, recherche les places élevées ». Cet exode rural se traduit par l'apparition de ce qu'il est convenu d'appeler une "population flottante ", c'est-à-dire de personnes qui, selon leur livret de résidence (hukou), devraient habiter à la campagne mais qui, en réalité, vivent en ville. D'après le recensement de 2010, cette population flottante peut être estimée à 300 millions, soit près d'un Chinois sur cinq.

Cette situation résulte des différents flux migratoires enregistrés au fil des recensements. Les données fournies par le Bureau national de la statistique de Chine (BNSC) montrent que les migrations internes totales ont concerné 261 millions de personnes entre les recensements de 2000 et de 2010, contre 144 millions entre 1990 et 2000. La majorité de ces migrants ont quitté les campagnes pour rejoindre des villes tout en restant au sein de leur entité administrative d'appartenance. Environ un tiers ont changé d'entité administrative, soit 42 millions entre 1990 et 2000 et 86 millions entre 2000 et 2010. La hausse des migrations entre entités administratives a donc été aussi importante que celle des migrations 


\section{Graphique 1. La montée des migrations internes en Chine}

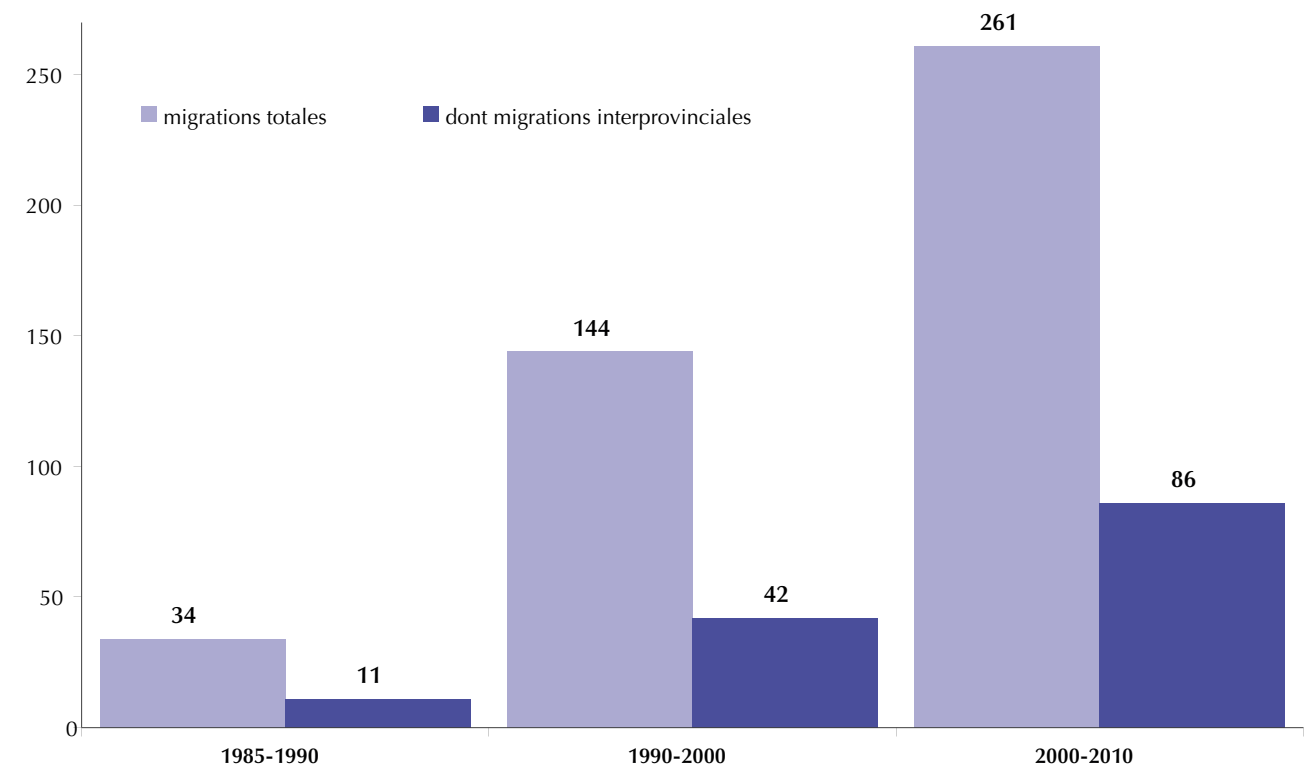

(C) Dumont G.-F., chiffres BNSC en millions d'habitants.

internes à celles-ci. Cette double hausse a engendré une très forte croissance du taux d'urbanisation : alors qu'il avait diminué entre 1965 et 1975, passant de $18,1 \%$ à $17,4 \%$ de la population totale, depuis le début de I'application des réformes entamées en 1978, il est remonté à près de $20 \%$ en 1980 et concernait près de la moitié de la population en 2010. L'émigration rurale a donc été massive.

\section{Une "population flottante » défavorisée}

La pauvreté est un élément explicatif central de cette émigration rurale puisque celle-ci se révèle particulièrement importante dans des régions pauvres comme le Sichuan ou le Hunan. Ces provinces essentiellement rurales constituent en quelque sorte, compte tenu de l'importance de leur peuplement, des réserves de population pour satisfaire les besoins de maind'œuvre des provinces en forte croissance économique. L'émigration rurale se dirige principalement vers des territoires à meilleur niveau de développement pouvant offrir des possibilités d'emploi ou de formation à des personnes sans qualification ou peu qualifiées.

La population flottante appartient aux catégories socio-

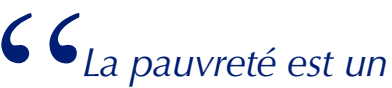

élément explicatif central de [l']émigration rurale (...). professionnelles les moins favorisées. Elle comprend d'abord les travailleurs migrants saisonniers, appelés mingong. Ils font penser aux oiseaux migrateurs, se déplaçant selon les mois de l'année pour trouver du travail ; ils peuvent ainsi être employés dans une région lors de la saison de la cueillette du coton, puis dans une autre pour la saison des récoltes agricoles, et ensuite dans une ville à la période la plus intense des 
constructions d'immeubles. L'autre composante de la population flottante travaille dans des métiers à faible qualification : dans la construction, de petites activités commerciales ou des activités de services comme la réparation de bicyclettes, les salons de coiffure, les restaurants et les hôtels, le ménage ou la garde d'enfants.

La population flottante se caractérise par de moins bonnes conditions sociales que les urbains domiciliés en ville. Ceux-ci l'acceptent souvent mal, la rendant responsable de l'augmentation de la criminalité ou de la surcharge des infrastructures urbaines qui n'arrivent pas à suivre le rythme de la rapide croissance du taux d'urbanisation. On reproche aussi souvent à la population flottante d'avoir une prévalence élevée du sida, liée à l'utilisation de seringues pour la consommation de drogue ou à l'exercice de la prostitution.

En 2003, le gouvernement central chinois a bien lancé le «nouveau paradigme pour le développement », une politique destinée à améliorer le bienêtre des immigrants ruraux qui vivent dans les villes. Mais la plupart des gouvernements locaux sont loin d'avoir mis pleinement en place cette politique. Ainsi, bien que la loi prévoie d'améliorer I'accès à la santé et au logement, I'assurance au travail et le droit à la retraite pour la population flottante, ces objectifs ne sont pas atteints, d'autant moins qu'il n'y a guère de contrôle ni d'évaluation de la mise en œuvre de cette nouvelle politique.

\section{L'importance des stratégies matrimoniales}

Une autre cause de l'émigration rurale tient aux stratégies matrimoniales, qui pèsent d'autant plus que la Chine est un pays où le mariage est une norme très forte et doit intervenir à un âge relativement jeune. Par exemple, des parents vivent mal le fait que leur fille de 25 ans ne soit pas mariée. Ainsi, parmi les émigrants des provinces du Yunnan ou du Guizhou, $50 \%$ des femmes émigrent par mariage vers des provinces comme le Hebei, le Fujian, le Anhui ou le Jiangsu, où les hommes sont majoritaires en raison de la politique de l'enfant unique et de la préférence familiale pour le garçon. Les jeunes actifs de sexe masculin peu qualifiés ayant le statut de résident dans les grandes villes, qui ont peu de chance de trouver une épouse en ville, peuvent ainsi se marier. Les femmes du monde rural sont moins regardantes sur la qualification de leur futur mari du moment que le mariage leur permet d'obtenir le droit de vivre en ville, officialisé sur le livret de résidence. Quant aux hommes de provinces riches, comme le Fujian, ils ont les moyens de rémunérer des agences matrimoniales pour se procurer une femme dans des régions plus pauvres de la Chine comme le Guizhou. Le fort déficit de filles dû à la politique de l'enfant unique crée des inégalités territoriales. Du fait du départ de femmes vers des provinces plus riches ou vers les villes pour 
Carte 2. Les flux migratoires entre les entités administratives chinoises entre 2000 et 2010

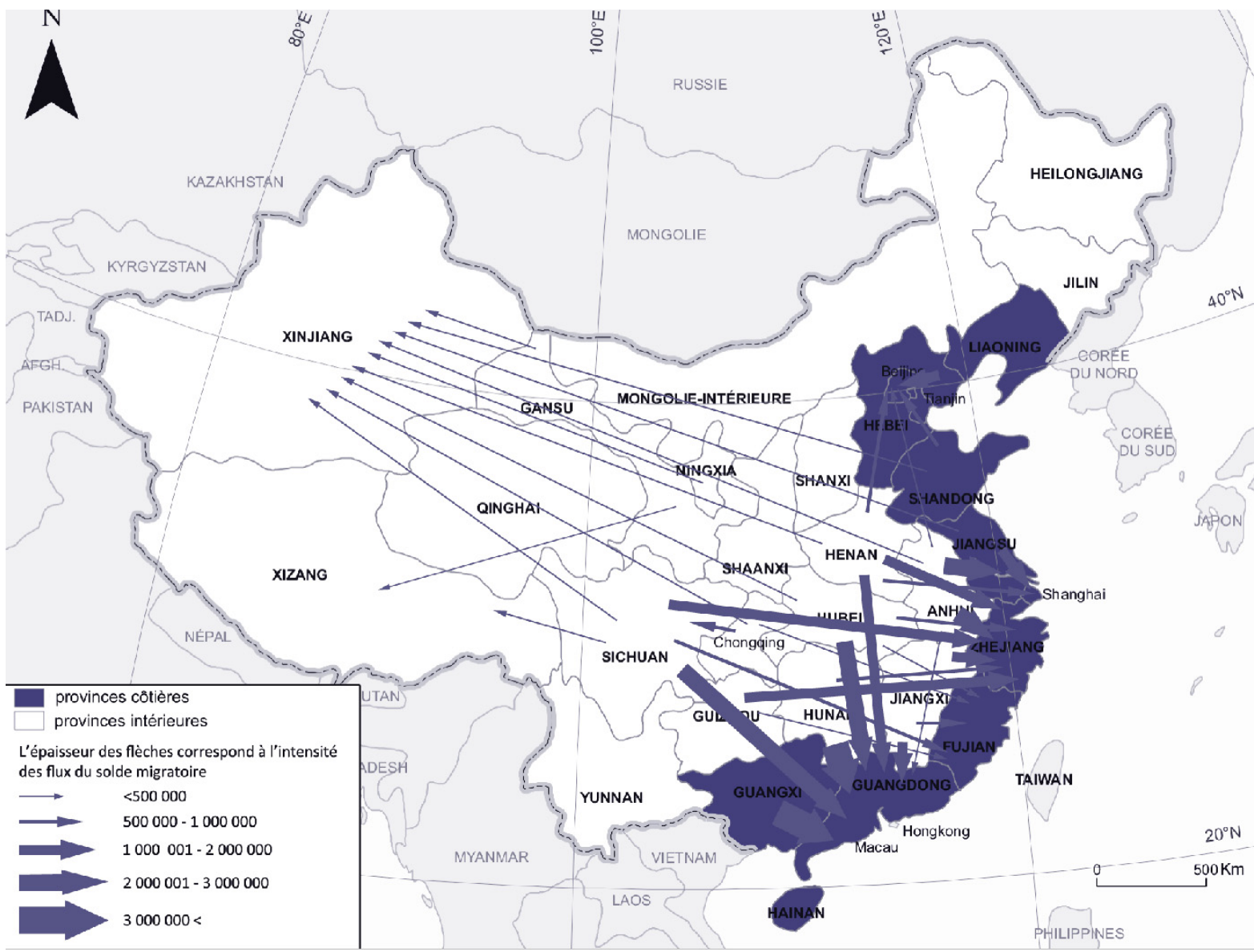

(C) Population et Avenir, chiffres BNSC 2012.

obtenir le droit de résider en ville par mariage, les régions rurales subissent par ricochet un déficit de filles accentué.

Une autre catégorie d'émigrants ruraux est constituée de jeunes couples qui partent en ville pour trouver des revenus professionnels. Ils confient alors leur(s) enfant(s) à leurs parents restés dans le monde rural où ils travaillent dans l'agriculture. On constate parfois des troubles psychologiques chez ces enfants privés de leurs parents, en dehors des visites que font ceux-ci pour des fêtes comme le Nouvel An.

\section{Une situation sociale duale}

La disparité du peuplement en Chine est certes historique. Mais la différence de statut entre la population rurale et la population urbaine crée une situation sociale duale pour les Chinois. La population vivant dans les campagnes ou formant la population flottante des villes ne bénéficie pas du même régime de protection sociale que celle ayant un permis 
de résidence en ville, en raison de ce qu'on appelle le régime de la " collectivité rurale » et le "système d'enregistrement de l'état civil» (hukou). Si la réforme économique entamée en 1978 a généré une remarquable croissance économique en Chine, elle a, dans le même temps, accru les disparités régionales. En outre, il existe un autre problème social lié aux migrations internes : la migration de Hans, fortement encouragée par les autorités de Pékin vers les régions dites « autonomes », comme le Xinjiang ou le Xizang (Tibet), a exacerbé les tensions interethniques. Lorsque le gouvernement accorde des avantages aux immigrants pour faciliter leur implantation, la population locale, en particulier paysanne, se trouve injustement traitée. La cohésion sociale qui devrait régner au sein d'un pays qui vise une société harmonisée se trouve fragilisée par diverses disparités territoriales qui se sont accentuées ces dernières décennies.

\section{Note}

1 - Nous laissons de côté les deux entités administratives spéciales de Hong Kong et de Macao, dont la superficie est trop réduite pour entrer dans le cadre de notre analyse.

\section{Bibliographie}

- BNSC (Bureau national de la statistique de Chine), 2013, Zhongguo tongji nianjian-2013 (China statistical Yearbook-2013), Pékin, China Statistic Press ; 2012, Zhongguo 2010 nian renkou pucha ziliao (Données du recensement de 2010 en Chine), Pékin, Éditions de statistique de Chine ; 2002, Zhongguo 2000 nian renkou pucha ziliao (Données du recensement de 2000 en Chine), Pékin, Éditions de statistique de Chine.

- Dumont G.-F., 2013, « Démographie du Tibet. Quels scénarios géopolitiques?" Diplomatie, n 63, p. 40-44; 2011, "Les six enjeux de la démographie chinoise ", Géostratégiques, n 33, p. 17-43; 2008, "Le déficit des filles en Chine : vers un nouvel enlèvement des Sabines? ", Monde chinois, $\mathrm{n}^{\circ} 15$, p. $57-66 ; 2004$, "Chine : des politiques coercitives", Informations sociales, $\mathrm{n}^{\circ} 115$, p. 16-17.

- Shen W. et Feng X., 2013, "Chengli de nongcun xifu : nongcun nvxing hunyin de chengshi shiying " (Recherche sur l'adaptation à la vie urbaine des femmes immigrantes par le mariage), Journal de l'Institut pédagogique de Hunan, $\mathrm{n}^{\circ} 2$, p. 84-90.

- Xiong L., Luo H., Li J. et Hu Y., 2005, « Behavior characteristics, spatial transitions of floating population and HIV/AIDS spread", Population \& Economics, n 6, p. 6-10.

- Yuan X., 2009, "Xin Zhongguo Renkou 60 nian » (La population de la Chine depuis soixante ans), Population Research, vol. 33, n 5, p. 42-67. 